\title{
Building capacity for astronomy research and education in Africa
}

\author{
Peter Martinez ${ }^{1}$ \\ ${ }^{1}$ South African Astronomical Observatory, P.O. Box 9, Observatory 7935, South Africa \\ email: peter@saao.ac.za
}

\begin{abstract}
About 1.5\% of the world's professional astronomers are based Africa, yet in terms of research output, African astronomers produce less than $1 \%$ of the world's astronomical research. The advent of new large-scale facilities such as SALT and HESS provides African astronomers with tools to pursue their research on the continent. Such facilities also provide unprecedented training opportunities for the next generation of African astronomers. This paper discusses recent efforts to develop astronomy education and research capacity on the continent. Various capacity-building initiatives are discussed, as well as the lessons learnt from those initiatives.
\end{abstract}

Keywords. Astronomy, Africa, education, research

\section{Introduction}

In his survey of astronomy around the world, Heck (2000) presented geographical distributions relating to various aspects of astronomy world-wide. The striking feature in all Heck's maps is what he described as "the desperate emptiness of most of the African continent." Of the 52 countries in Africa, only nine (viz: Algeria, Egypt, Ethiopia, Kenya, Libya, Mauritius, Morocco, Nigeria and South Africa) were listed by Heck as having some astronomy-related organizations. No other region of the world has such a dearth of activity in astronomy. A closer look at the nature of the facilities in these countries reveals that only Egypt, Namibia, Mauritius and South Africa and possess operational large-scale astronomical research facilities.

Professional astronomy in Africa is dominated by Egypt in the north and South Africa in the south. South Africa invests more in astronomy annually than all other African countries combined. This is reflected in the scientific output, which is greater than that of all other African countries combined - by a wide margin. The historical development of astronomy in South Africa, up until 1994, has been reviewed by Feast (2002). Whitelock (2004) reviewed developments in post-apartheid South Africa, from 1994 to 2004.

In this paper we will take a closer view of the status of professional astronomy in the rest of Africa and we will review some of the lessons learnt from various capacitybuilding initiatives that have taken place in recent years. This study encompasses the 46 countries in continental Africa and the independent island states Cape Verde, Comoros, Madagascar, Mauritius, Sao Tome and Principe and Seychelles - a total of 52 countries.

The focus of this study is on the development of professional astronomy at universities and research establishments. We do not include astronomy activities conducted purely in the amateur sphere, important though they are. Our reason for maintaining this distinction is that the pursuit of astronomy as a scientific discipline in a country requires a much higher level of professional and political commitment than the popularization of astronomy. Indeed, virtually all of the institutions involved in astronomy teaching or research in Africa are also highly active in the popularization of astronomy, but that is not their raison d'être. 
Table 1. Table 1: IAU membership statistics for Africa as of September 2006. Source: IAU website www.iau.org

\begin{tabular}{lrrrrrrr}
\hline & \multicolumn{2}{c}{ Members } & \multicolumn{3}{c}{$\begin{array}{c}\text { \% Members } \\
\text { National }\end{array}$} & \multicolumn{2}{c}{$\begin{array}{c}\text { Members } \\
\text { All IAU }\end{array}$} \\
National Members & Male & Female & Total & Male & Female & Male & Female \\
\hline Egypt & 51 & 6 & 57 & 89.47 & 10.53 & 0.52 & 0.06 \\
Morocco $^{1}$ & 7 & 0 & 7 & 100.00 & 0.00 & 0.07 & 0.00 \\
Nigeria & 9 & 1 & 10 & 90.00 & 10.00 & 0.09 & 0.01 \\
South Africa & 58 & 6 & 64 & 90.63 & 9.38 & 0.59 & 0.06 \\
\hline
\end{tabular}

Individual Members

\begin{tabular}{llllllll}
\hline Algeria & 3 & 0 & 3 & 100.00 & 0.00 & 0.03 & 0.00 \\
Ethiopia & 1 & 0 & 1 & 100.00 & 0.00 & 0.01 & 0.00 \\
Mauritius & 1 & 0 & 1 & 100.00 & 0.00 & 0.01 & 0.00 \\
\hline
\end{tabular}

Total Members

\begin{tabular}{lrrrrrrr}
\hline All Africa & 130 & 13 & 143 & 90.91 & 9.09 & & \\
All IAU & 8451 & 1322 & 9773 & & & 86.47 & 13.53 \\
\hline
\end{tabular}

Notes:

${ }^{1}$ Morocco has interim membership status.

\section{Organized astronomy communities in Africa}

A good way to depict the presence of organized astronomy in Africa is to consider membership of the International Astronomical Union (IAU). Table 1 depicts the IAU membership statistics for Africa as of September 2006. The total African membership of the IAU amounts to 143 persons in 7 countries, corresponding to $1.5 \%$ of the total IAU membership. The African membership is dominated by South Africa (45\%) and Egypt (40\%), followed by Nigeria (7\%) and Morocco, Algeria, Ethiopia and Mauritius, all with $5 \%$ or fewer members. It is also instructive to compare IAU membership changes in recent years. IAU membership has increased by $13 \%$ from 127 to 143 during the past triennium. This reflects the growth in astronomical communities in South Africa and Nigeria. The membership numbers in the other countries have remained static during this period.

The dearth of IAU adhering countries in Africa is striking. The only adhering countries in Africa are Egypt, Morocco (interim status), Nigeria and South Africa. Fortunately the IAU's rules are flexible enough to permit individual $\mathrm{PhD}$-qualified astronomers to join the IAU in their personal capacity, even if their countries are not yet ready to join as national members. Through this mechanism, individual astronomers in Algeria, Ethiopia and Mauritius are also members of the IAU. I believe it is very important to maintain this admissions policy as it reduces the isolation of scientists who return to their countries in Africa after obtaining their training in astronomy elsewhere.

One might ask, if it is possible for individual scientists to belong to the IAU, why should it matter that African countries have organized astronomical communities? The answer to this becomes apparent when one considers that the map of IAU member countries reflects the more prosperous African nations in a Gross Domestic Product (GDP) map of the continent. Economic prosperity in the information society is closely linked to the ability to harness science and technology as a "smart user." Virtually all countries are critically reliant on space applications these days. The basic space sciences and their supporting technologies underpin the ability of a country to utilize space applications programmes 
for development. Countries that belong to the IAU are countries that recognize the importance of astronomy as a national activity to their future well-being.

\section{Tracers of research activity in space science}

The IAU membership figures, though instructive, do not present the complete picture as far as the distribution of individual scientists in Africa because not all of them are members of the IAU, either through a national adhering organization or in their personal capacity. A better way to gauge the distribution of active individual space scientists is to examine the literature, and also the online usage of the astronomical literature.

Martinez (2006) discussed an analysis of the literature archived in the NASA Astrophysics Data System (ADS) database of abstracts for the period 1973-1996. Out of a total of 181808 papers in the ADS data base, 1339 ( $0.74 \%$ ) had a principal author or at least one co-author affiliated to an institute in an African country. The top five countries identified in this study were South Africa, with $77 \%$ of the African papers, followed by Egypt (14\%), Nigeria (4\%) and 19 other countries with fewer than 1\% of the papers. It is not surprising that the top five countries in this list are also the top five countries by GDP in Africa. This sample is very incomplete, and serves only to indicate that researchers producing papers relating to some aspect of astronomy are more widely spread throughout Africa than a simple inspection of IAU membership statistics would suggest. These are the countries that IAU Commission 46 can reach out to in the future.

Recent enhancements to the query forms in the ADS make it possible to query the ADS on-line for affiliations by country. However, comparison of the results of such a search with the publication statistics for South Africa, which are well known to the author, indicate that this type of query returns an incomplete sample at best. Moreover, the results are contaminated with papers that pertain to Earth observation or to nonastronomical topics in physics. Thus, ADS publication counts are at best a noisy data set with which to study astronomical research activity in Africa. Their value lies in revealing the isolated scientists that are active in research as potential local partners for future capacity-building interventions.

A more reliable metric of current astronomical research activity is to examine the country of origin of accesses to the ADS data base. As of this writing, a project is in progress to record these accesses on a month-by-month basis. The early results are encouraging. In October 2003, the Working Group on Space Sciences in Africa published a special issue of African Skies / Cieux Africains on the ADS, including remote access by email (Eichhorn et al. 2003). Subsequent to the publication of that issue, a number of African countries started to access the database (Fig. 1). As internet connectivity in Africa improves, and as more African astronomers become aware of the ADS, we may expect that the ADS will see greater usage by African astronomers. This is an absolutely critical service for the development of professional astronomy in Africa, as most African institutions do not have astronomical libraries. The ADS is a major contributor towards reducing the isolation of African scientists.

Kurtz et al. (2005) analysed the utilization of the ADS data world-wide as a function of GDP and they found a simple relation that ADS use per capita is proportional to GDP per capita squared. Figure 3 of Kurtz et al.'s paper is instructive. They plot the number of ADS queries per million inhabitants versus GDP per capita. The only African countries to appear in their plot are Egypt, Morocco, and South Africa, which appear to conform to the relation

$$
(\text { ADS use }) \propto(G D P \text { per capita })^{2}
$$




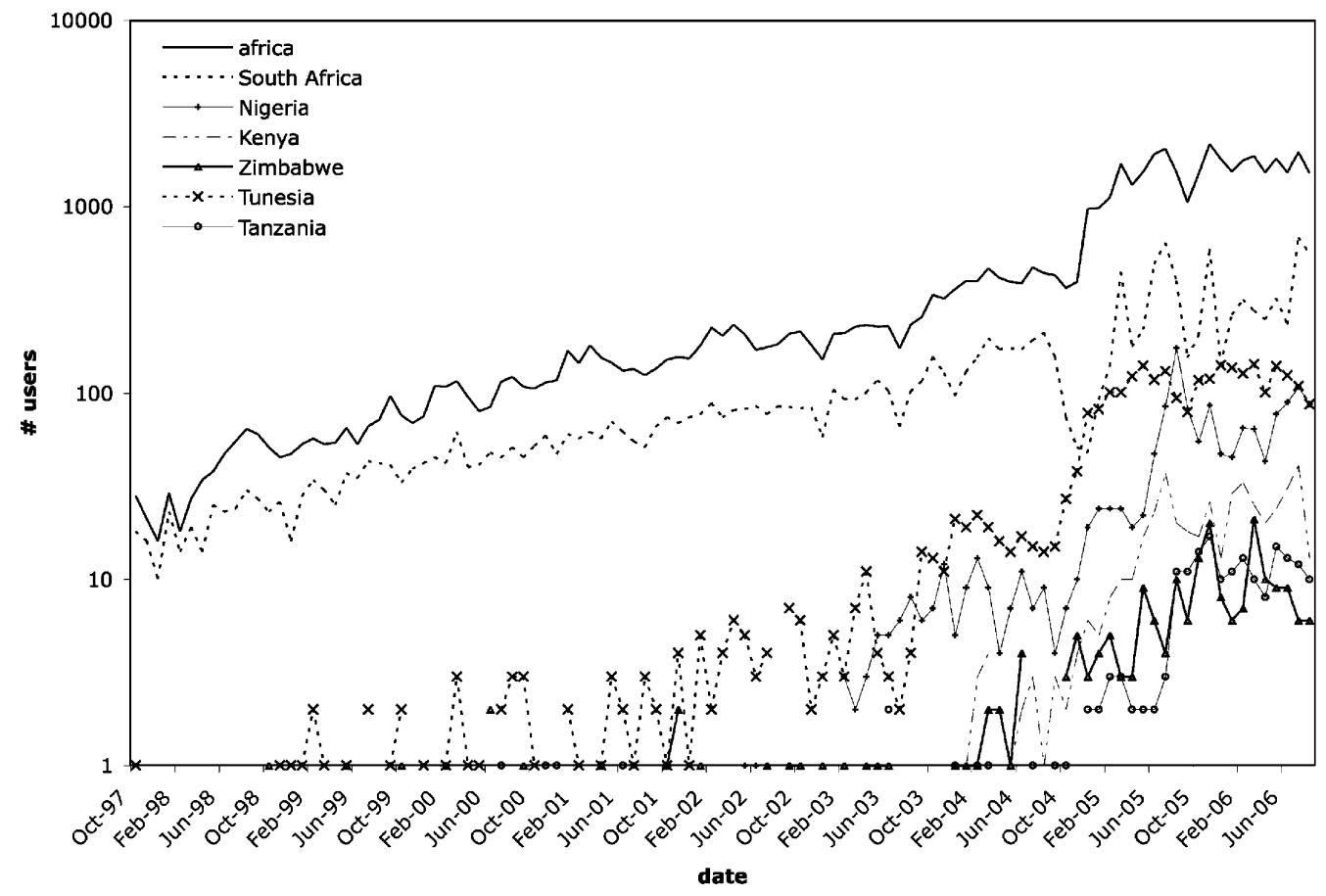

Figure 1. Usage of the NASA ADS by scientists in African countries. Figure courtesy of Guenther Eichhorn, ADS.

It is not surprising that these are also three of the four IAU Member countries in Africa.

\section{New large-scale facilities}

Although the prospects for astronomy in the continent as a whole are not encouraging, there are some notable improvements compared to the situation as it was ten years ago. The improvements are most noticeable in the southern African region, where political stability, government support for science, and excellent astronomical conditions have attracted a number of large-scale international astronomical facilities. Namibia hosts the international High Energy Stereoscopic System (HESS), an array of four imaging atmospheric Cherenkov telescopes for the investigation of cosmic gamma rays in the 100 GeV energy range (Hinton, 2004). HESS is the premier facility of its kind in the world. The Southern African Large Telescope (SALT) is a $10-\mathrm{m}$ optical telescope inaugurated in November 2005 by South Africa and 10 international partners. SALT is currently the largest single optical telescope in the southern hemisphere. South Africa is also currently developing the Karoo Array Telescope (KAT), a 1\% technology demonstrator of the Square Kilometre Array (SKA), which South Africa is bidding to host. In addition to these large-scale facilities a number of smaller robotic telescope facilities are coming online, which may be accessed remotely over the internet by scientists in Africa (see for example Martinez et al., 2002). For the first time, these facilities provide an opportunity for African scientists to perform cutting-edge research on the continent and in the context of their own national environments. The challenge is to create the right conditions to attract and retain bright young scientists in their own national environments after they complete their studies. 


\section{Capacity building initiatives - lessons learnt}

Over the years, a number of institutions and international organizations have conducted capacity building activities to develop astronomy in Africa. The IAU supports capacity building in Africa principally through the activities of its Commission 46, Astronomy Education and Development. Activities within this Commission are organized into nine Programme Groups (Isobe 2003). The capacity-building activities of COSPAR are conducted through the COSPAR Panel on Capacity Building. This Panel has conducted a series of workshops in developing countries aimed at increasing the utilization of space archive data (Arnaud \& Willmore, 2006 - this volume). The first such workshop in Africa was held in Durban, South Africa, in July 2004 on the topic of X-ray astronomy. For many years, the United Nations Office for Outer Space Affairs has been arranging the UN/ESA Workshops on Basic Space Science (Haubold \& Wamsteker 2004) which have been attended by a considerable number of African scientists.

So, what have all these activities amounted to? Why is there still a dearth of astronomy research and training activities in Africa? There are no simple answers to these questions. However, it is possible to distill out of my experiences of capacity building in a variety of African countries over the past decade a series of lessons learnt and some basic conditions which must be fulfilled before astronomy can thrive in a country.

\section{Seek out fertile ground in which to plant a seed}

By "fertile ground" I mean several things. Firstly, an adequate infrastructure is a sine qua non of sustainable capacity building for research in astronomy. This includes things such as computers, internet access, library facilities, and so on. $\dagger$ Secondly, this must be matched by an institutional environment with a supportive hierarchy. Without committed institutional support, there is no hope of a capacity-building initiative becoming sustainable in the long run as it will collapse the moment the external support ends or the key person(s) withdraw for any reason. Thirdly, there should be cohesion and a common unity of purpose within the developing scientific community for capacity building initiatives to take root. Fourthly, there should be a supportive (or at least non-obstructive) national environment. For example, in a supportive environment, there are means to remove or minimize administrative and fiscal impediments such as import duties on donations of scientific equipment or books.

\section{Capacity building is about people, not about equipment}

Don't begin a capacity building programme with the installation of an observatory before the following are in place: (i) a clear scientific plan for how to utilize the telescope, (ii) a clear technical plan to support the telescope, (iii) adequately trained people in place. A capacity-building initiative that is based around equipment, rather than around people is doomed to fail. When contemplating the installation of new facilities as part of a capacity-building programme, it is important to ensure that the necessary human capital is developed through training in the operation, purposeful use and maintenance of equipment, before such equipment is put in place. Given the limited resources available for capacity building, an approach that works well is to train the trainers. We have used this approach to introduce astronomy into the undergraduate physics curricula at various African universities, and we are starting to see more African students enrolling for postgraduate degrees in astronomy on the continent and elsewhere.

$\dagger$ Note that I did not include a telescope in this list. This is not because I believe that telescopes are unimportant, but here we are talking about a basic infrastructure for research. Without these elements in place, a telescope will not be scientifically productive. 


\section{Invest in young people}

Capacity-building opportunities should target young people who are not burdened with administrative or other duties and have more time to drive developments from the bottom up. The same lesson applies also to the scientists doing the capacity building. Many young professionals are keen to share their expertise with colleagues from developing countries and they generally have the mobility and time to do so. The challenge to the older scientists in developed and developing nations is how to engage most effectively with the capacity-building process in such a manner as to allow their younger colleagues to achieve the desired sustainable results.

\section{Capacity building is a process, not an event}

Capacity building activities must be part of a long-term programme. Activities such as capacity building workshops will not lead to sustained research activity if they are not part of a long-term programme. Hence the importance of strategic partnerships among the scientific unions, the scientific community in the developing country and the development aid sector. It normally takes several years of engagement to build up some level of sustainable activity. This requires firm commitment from all partners in the face of occasional setbacks and failures, even in the best supported scenarios.

\section{Succession planning is important}

The developing world is replete with examples of small groups that flourished under the guidance of an inspired individual for a period, and then faded out of existence when that person left, retired or passed away. When a new capacity-building initiative starts, it is not unusual for it to be championed by the senior academics or scientists, who have the managerial experience and academic credentials to obtain support from their university authorities or government. However, these same scientists have a career investment in an area outside of astronomy and are never likely to have the time to become independently competitive astronomers themselves. But they will inspire their students to study astronomy at postgraduate level, and it is those students who will one day return to establish a solid, independent astronomy research group. It is thus very important to develop a succession plan as part of the long-term capacity building strategy. This gives the institution some guarantee of continuity, but at the same time it also entails institutional commitment to the young scientists, who know that there will be a position to return home to. This is a fundamental aspect of the commitment to astronomy that we discussed in Section 2.

\section{Match new facilities to education and research needs}

Often capacity building in astronomy focuses around the acquisition of a small telescope. Small $(<0.5 \mathrm{~m})$ telescopes have an important role to play in undergraduate teaching and student training. Regular access to such telescopes by students and the public can do much to promote astronomy in a developing country. However, above about $0.5 \mathrm{~m}$ aperture I believe one needs to consider whether investing in a telescope is the best way to promote internationally relevant astronomical research in a particular environment. For many African countries, I would argue that a good internet connection and access to large-scale facilities elsewhere is far more likely to result in productive research than an ill-equipped telescope at a poor site.

\section{Astronomers should not pretend to be engineers}

This may seem an obvious statement, but I have seen many instances where astronomers try to perform functions that engineers are trained for. The result is that they spend all their time fussing over equipment that never quite performs to specification, and they 
have no time to do any science. Moreover, the students who struggle with such equipment often lose their motivation to study astronomy, or, worse, wind up learning very little astronomy. A small observatory with very limited resources should consider whether its priority is to do astronomy, or to do engineering. If astronomy is the priority, it might be better to acquire a professionally built research-grade telescope and/or instruments, rather than trying to develop this in-house. If this is not a possibility, then it would be better to obtain service observations from telescopes at good sites or to use data from space missions.

\section{Use information technology as much as possible}

Information technology is a powerful enabler of research. Access to email and online literature, software and data reduces isolation of scientists and makes them much more productive. For the developing world Open Source is a particularly enabling technology in the sense that, in addition to the cost savings associated with keeping software current, the open nature of the software leads to greater innovation and allows users to adapt it to local demands and steer their own IT infrastructure. Moreover, because the astronomy community is a heavy user of Open Source software, the skills acquired by participants in capacity-building activities can be of benefit not only to themselves, but also to their home institutions.

In the astrophysics domain, most of the literature is available on-line through the NASA Astrophysics Data System (Eichhorn, 2004), and large quantities of astrophysical data are available on-line or on request from a variety of data centres. This provides excellent opportunities to promote research in developing nations without needing to develop costly infrastructure in conditions that are sub-optimal for ground-based astronomy. Moreover, the same IT infrastructure that is required to do cutting-edge astrophysical research can also be utilized for other purposes, so the benefits of the investment are enjoyed by a much wider community of users than just the astronomers.

Capacity building activities should also take into account the general level of preparation of participants. For example, "hands-on" capacity building activities assume a certain degree of computer literacy on the part of the participants. However, one cannot assume familiarity with the computing environments used in astrophysics. Often a carefully planned training activity is compromised when time is lost bringing people up to speed with very basic computer skills. Better screening of prospective participants and/or precursor computer training would enhance the efficacy of such activities in future. Attention also needs to be given the the participants' home computing environments. It does no good to train people to use software tools that cannot run on computers at their home institutions. In this case, perhaps the provision of computers needs to be part of the capacity-building plan. This is where development aid agencies come into the picture.

\section{Promote and nurture regional initiatives and facilities}

Regional networks represent the scientific community's determination to organize itself and its activities. Close cooperation between the scientific unions and these regional structures can be mutually beneficial and support sustainable capacity building. Regional networks, such as the Working Group on Space Sciences in Africa, can support capacity-building initiatives of the scientific unions by organising or promoting awareness of key events, such as workshops, and by supporting the follow-up phase afterwards. Regional networks can also assist scientists with accessing facilities in the region and with 
accessing training and career opportunities on the continent. Africa has two regional centres affiliated to the United Nations for training in space science and technology, one in Nigeria for anglophone Africa and one in Morocco for francophone Africa. These centres form an important part of the constellation of facilities available on the continent for human resource development. Though neither centre has a component of astronomical research at the moment, such a component could be developed in partnership with astronomy institutes elsewhere in the region.

\section{Focus on solving real research problems}

Capacity building initiatives (visits, workshops) often focus on equipping the participants with the skills and tools to conduct research in a given field, yet few participants go on to initiate research projects on returning to their home institutions. I believe the reason for this is that these scientists work in isolation, with no idea of what are the relevant problems to tackle. One way to address this is to structure the capacity building activity around some area of research that the group of participants can continue to work on as a network after they return to their home institutions. Establishing networked collaborations is a good way to build up a critical mass of scientists who can support each other and produce science that is relevant and of international quality. This will require ongoing support and encouragement from colleagues in developed countries, but will yield a piece of publishable research and take the group of participants through the whole research cycle.

\section{Form partnerships for capacity building}

Working in partnerships allows an organization to leverage its resources with those of other organizations and to coordinate collective efforts for maximum impact. Working in partnership also introduces complications. The different organizations will have their own objectives, programmes and financial cycles, but they are more likely to support initiatives that are supported by other partners as well. The types of interactions that are most likely to lead to sustainable capacity building are those that also attract the support of the development sector and/or government. A good example of this is the COSPAR/IAU Regional Workshop on Data Processing from the Chandra and XMMNewton Space Missions, held in Durban, South Africa in July 2004. This workshop arose from cooperation between the capacity building programmes of the IAU and COSPAR discussed earlier in this paper, with additional funding from the National Research Foundation of South Africa, the UN Office for Outer Space Affairs, the Abdus Salam Centre for Theoretical Physics and the European Space Agency.

\section{Closing remarks}

With the advent of new large-scale facilities for ground-based astronomy in Africa, such as the Southern African Large Telescope (SALT) and the High Energy Stereoscopic System (HESS), and a regional climate of enhanced scientific cooperation, African astronomers are now able to access to some of the world's premier astronomical facilities without having to leave the continent. Increasing internet penetration and access to the literature through on-line services such as the NASA ADS is reducing the scientific isolation of African astronomers. Together, these factors should lead to a steady growth of astronomy on the continent. Indeed, IAU membership over the past triennium in Africa has increased by $13 \%$. This growth is encouraging, but it will have to be sustained for many years to build a significant and vibrant astronomical community on the continent. 
The development of new facilities in Africa is largely the result of investment by the international community in Africa. In the southern hemisphere the continent has some of the world's best astronomical sites, free of light pollution and radio interference. African countries are beginning to recognize the geographical advantages of the region and are seeking to develop the region as a southern hemisphere hub of astronomy. South Africa is in the process of enacting very progressive legislation to protect present and future astronomical sites from activities that might degrade the astronomical conditions across the electromagnetic spectrum. The region as a whole is backing the South African-led bid to host the SKA on the continent. These are encouraging indications that astronomy is flourishing in at least some parts of the continent.

Developing astronomical research and education capacity in Africa is a long-term activity. The IAU, COSPAR and the UN have played a very significant role through their activities on the continent over the past few decades. The actions of these role-players have all had some long-term effect on individuals, and the cumulative impact of these interventions will take years to manifest themselves. The participants in these activities have often returned home to inspire their students, who in turn aspire to astronomical careers and pursue their studies at major astronomical centres. The capacity-building efforts of organizations such as the UN, IAU, COSPAR and the Working Group on Space Sciences in Africa are thus starting to yield fruit in terms of producing a new generation of African astronomers. Working in partnerships greatly enhances the impact of capacity-building activities. In order to be sustainable, capacity-building activities should form part of a comprehensive long-term programme. Such a programme should address pipeline issues to ensure that interventions by the different role-players are mutually supportive and appropriately phased. In order to facilitate coordination of activities by the different organizations, consideration should be given to the establishment of a capacitybuilding forum. Such a forum could facilitate improved dialogue between the scientific unions and scientific institutes, the development sector (e.g. UNESCO) and the developing countries to link the players with the technical means (the scientific community) to the communities with the needs (the developing countries) through provision of support for development of infrastructure and operation of projects by the development sector. An organization like the Working Group on Space Sciences in Africa would be well placed to initiate such a development. The challenges are immense, but so, too, are the rewards.

\section{Acknowledgements}

I acknowledge with gratitude the generous support received from the following organizations for the various capacity-building initiatives described in this paper: Observatoire Midi Pyrénées, South African National Research Foundation, South African Astronomical Observatory, IAU, COSPAR, United Nations Office for Outer Space Affairs and UNESCO. I also acknowledge the contributions by many colleagues who have participated in the capacity building activities funded by these organizations, as well as the insights I have gained from working with them. This research has made use of NASA's Astrophysics Data System.

\section{References}

Arnaud, K., Willmore, A.P., in: J. Hearnshaw \& P. Martinez (eds.), Astronomy for the Developing World, Cambridge University Press, ..... This volume, pXX.

Eichhorn, G., Accomazzi, A., Grant, C.S., Kurtz, M.J., Murray, S.S. 2003, African Skies 8, 7

Eichhorn, G. 2004, Astron. and Geophys. 45:3, 7 
Feast, M.W. 2002, in: A. Heck (ed.), Organizations and strategies in astronomy III, Astrophysics and Space Science Library, Vol. 280. Dordrecht: Kluwer Academic Publishers, ISBN 1-40200812-0, p. 153

Haubold, H.J., Wamsteker, W. 2004, in: W. Wamsteker, R. Albrecht \& H. Haubold, (eds.), Developing Basic Space Science World-Wide: A decade of UN/ESA Workshops, Kluwer Academic Publishers, Dordrecht, p. 3

Heck, A. 2000, ApESS 274, 733

Hinton, J.A. 2004, New Astron. Revs 48, 331. See also http://www.mpi-hd.mpg.de/hfm/HESS/

Isobe, S. 2003, in: A. Heck (ed.), Organizations and strategies in astronomy IV, Kluwer Academic Publishers, ISBN 1-4020-1526-7, p. 189

Kurtz, M.J., Eichhorn, G., Accomazzi, A., Grant, C., Demleitner, M., Murray, S.S. 2005, J. American Soc. for Information Science and Technology 56:1, 36. Also available at http://cfa-www.harvard.edu/ kurtz/jasist1-abstract.html

Martinez, P., Kilkenny, D.M., Cox, G., et al. 2002, MNRAS 61, 102,

Martinez, P. 2006, in: A. Heck (ed.), Organizations and Strategies in Astronomy VI, Kluwer Academic Publishers, Dordrecht, p.39

Whitelock, P. 2004, in: A. Heck (ed.), Organizations and Strategies in Astronomy V, Kluwer Academic Publishers, Dordrecht, p.39

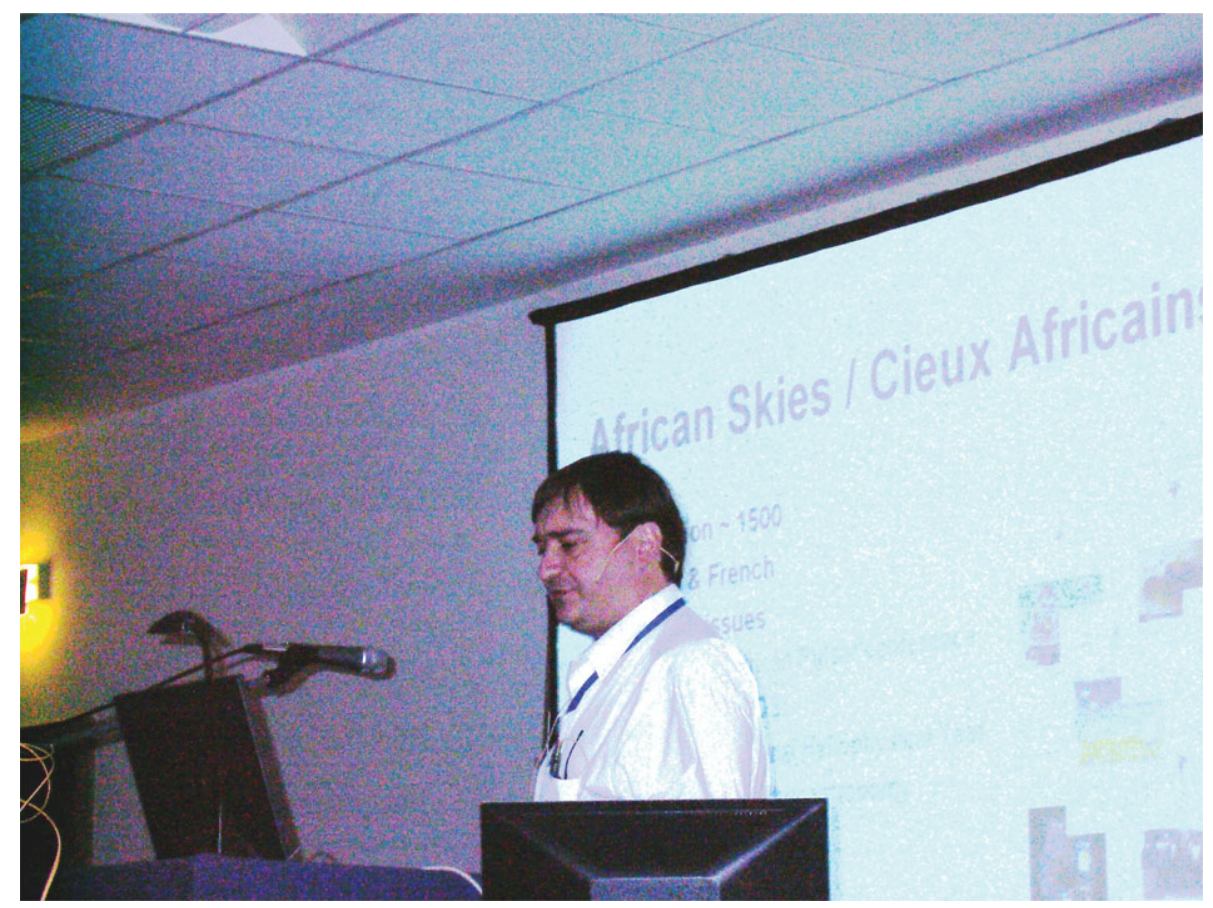

Peter Martinez 